\title{
COVID-19 in Newborns and Infants-Low Risk of Severe Disease: Silver Lining or Dark Cloud?
}

\author{
Munmun Rawat, MD ${ }^{1}$ Praveen Chandrasekharan, MD, MS ${ }^{1}$ Mark D. Hicar, MD, PhD ${ }^{1}$ \\ Satyan Lakshminrusimha, $\mathrm{MD}^{2}$ \\ ${ }^{1}$ Department of Pediatrics, University at Buffalo, Buffalo, New York \\ 2 Department of Pediatrics, University of California, Davis, California \\ Am J Perinatol 2020;37:845-849. \\ Address for correspondence Munmun Rawat, MD, Department of \\ Pediatrics, Oishei Children's Hospital, University at Buffalo, \\ Conventus, 1001 Main Street, Buffalo, NY 14203 (e-mail: munmun. \\ rawat@gmail.com).
}

\begin{abstract}
Keywords

- coronavirus

- COVID-19

- neonates

- SARS-CoV-2

One hundred years after the 1918 influenza pandemic, we now face another pandemic with the severe acute respiratory syndrome-novel coronavirus-2 (SARS-CoV-2). There is considerable variability in the incidence of infection and severe disease following exposure to SARS-CoV-2. Data from China and the United States suggest a low prevalence of neonates, infants, and children, with those affected not suffering from severe disease. In this article, we speculate different theories why this novel agent is sparing neonates, infants, and young children. The low severity of SARS-CoV-2 infection in this population is associated with a high incidence of asymptomatic or mildly symptomatic infection making them efficient carriers.
\end{abstract}

\section{Key Points}

- There is a low prevalence of novel coronavirus disease in neonates, infants, and children.

- The fetal hemoglobin may play a protective role against coronavirus in neonates.

- Immature angiotensin converting enzyme (ACE2) interferes with coronavirus entry into the cells.

The novel coronavirus disease 2019 (COVID-19) pandemic, first noted in Wuhan, China, during late 2019, has spread across the globe. Strikingly, initial reports suggest that neonates, infants, and young children are less affected by the disease (-Fig. 1).

As of April 18, 2020, the COVID-19 pandemic has resulted in approximately 2.3 million cases and over 157,000 deaths worldwide. In the United States, there have been 374,329 confirmed cases and 12,064 deaths. Twenty-two percent of United States population is less than 18 years of age. Among the 149,082 reported cases, only $2,572(1.7 \%)$ were children out of which $398(0.2 \%)$ were infants with $<1$-year age and a total of three deaths have been reported. ${ }^{1}$

This paralleled the trend that was seen in China, where out of over 80,000 cases, approximately 2,000 (2.5\%) were under 18 years of age and 379 were infants (0.4\%).
Over $90 \%$ of all patients were asymptomatic, mild, or moderate cases. $^{2}$

Human coronaviruses were discovered in the 1960s by researchers studying the etiology of upper respiratory infections (URIs) in children and young adults. The human coronaviruses cause 15 to $35 \%$ of URIs depending on the specific year. ${ }^{3}$ Mostly respiratory tract infections affect pediatric and geriatric population more severely than the younger adults. Centers for Disease Control and Prevention (CDC) estimated the overall cumulative hospitalization rate from influenza was 68 per 100,000 population across all age groups in the United States and 94 per 100,000 in 0 to 4 year age group. A total of 162 influenza-associated deaths in children have been reported so far this season. ${ }^{4}$ The flu vaccine reduced children's risk of flu-related pediatric intensive care unit (PICU) admission by $74 \%$ during flu seasons. ${ }^{5}$ received

April 19, 2020

accepted

April 19, 2020

published online

May 7, 2020
Copyright $\odot 2020$ by Thieme Medical Publishers, Inc., 333 Seventh Avenue, New York, NY 10001, USA. Tel: +1(212) 760-0888.
DOI https://doi.org/ 10.1055/s-0040-1710512. ISSN 0735-1631. 


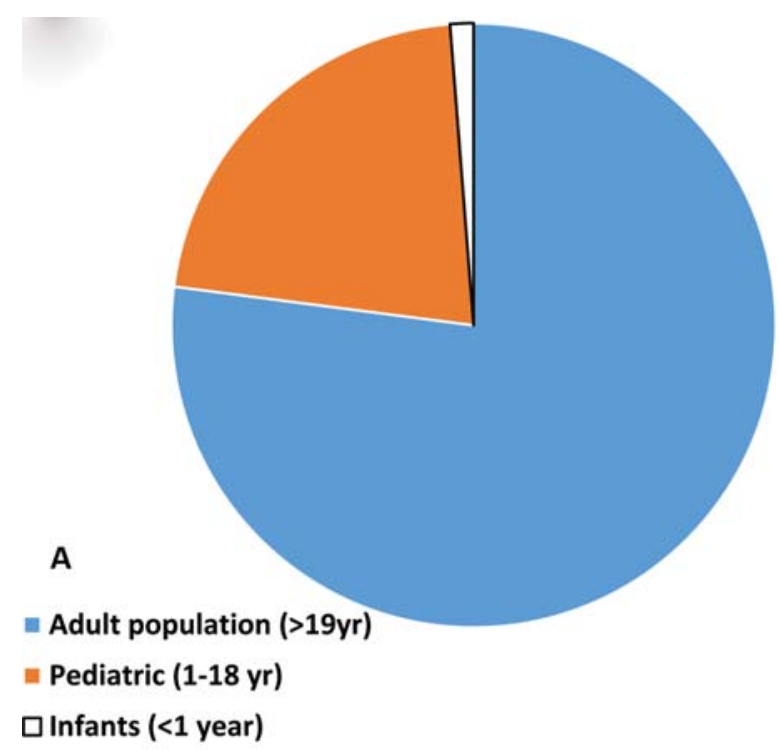

B

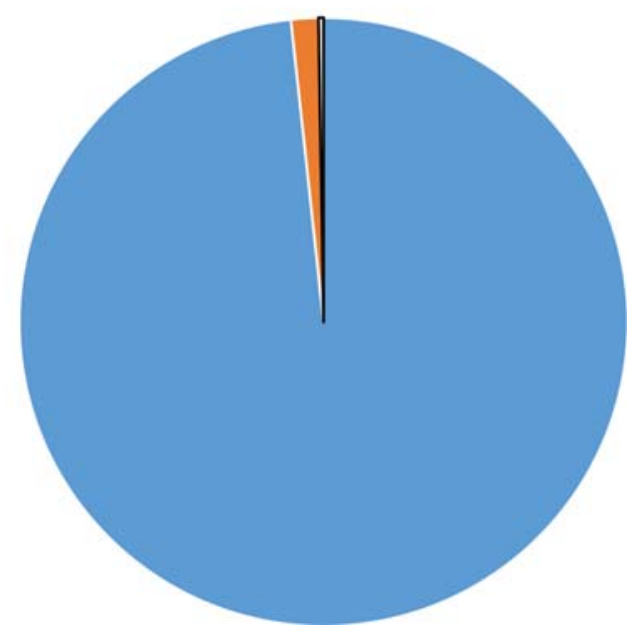

Fig. 1 (A) A pie chart showing distribution of population in United states based on age as adults ( $>19$-year olds), pediatrics ( $1-18$ years) and infants (<1-year olds). (B) The reported COVID-19 cases in adults, pediatrics and infant population in United States. Blue represents adults over 19-year olds, orange represents pediatric cases age $0-18$ year olds, and gray represents infants $<1$-year olds. COVID-19, novel coronavirus disease $2019 .{ }^{1}$

\section{Lessons Learnt from other Coronavirus- Associated Diseases: SARS and MERS}

Prior to the past two decades, the four circulating species of coronaviruses associated with human disease were known to be a significant cause of the common cold and other mild URIs. More severe lower respiratory infections (LRIs) were associated with the recent zoonotic crossovers of the severe acute respiratory syndrome (SARS)-associated coronavirus (SARS-CoV) identified in 2002 and Middle East respiratory syndrome coronavirus (MERS-CoV) identified in 2012.

A notable feature of the 2003 global SARS outbreak was the relative paucity of infections among children. In a published review of case series, the authors noted the absence of mortality in children and adolescents. In particular, children under 13 years of age had milder disease, no mortality, and only mild residual changes in exercise tolerance and pulmonary function at 6 months after resolution of illness. ${ }^{6}$ It was suggested that prior exposure to other respiratory viruses, making their immune systems more resilient, and inability to mount a mature immune response during the immune dysregulation phase of SARS and thus less organ damage was associated with low morbidity and mortality in children. ${ }^{7}$

As of November 2019, World Health Organization (WHO) reported a total of 2,494 laboratory-confirmed cases of MERS including 858 associated deaths (case-fatality rate: 34.4\%) globally. A review of pediatric cases through April 2016 revealed only 31 pediatric cases representing roughly $1 \%$ of cases known at the time. Only 18 were symptomatic and there was one death reported in a child with cystic fibrosis. The authors postulated a variety of explanations for lower case numbers in children including lower exposure rates, less symptomatic cases, and the presence of yet to be identified factors. ${ }^{8}$

\section{COVID-19: Why Are Young Children and Infants Not Severely Affected?}

Many theories have been postulated to explain this remarkable difference in the pediatric and adult population with COVID-19. Besides the various lifestyle factors (less overall and lifetime exposure to smoke or pollution, better nutrition, and more daily exercise), children have other specific advantages that might limit disease progression following SARSCoV-2 infection (-Fig. 2).

\section{Role of Angiotensin-Converting Enzyme 2}

Like SARS-CoV and coronavirus NL63, recent evidence indicates that entry of SARS-CoV-2 into cells requires the presence of angiotensin-converting enzyme 2 (ACE2) protein. ${ }^{9}$ ACE2 receptors are expressed in human airway epithelia, as well as lung parenchyma. Of note, undifferentiated cells expressing little ACE2 were found to be poorly infected with SARS-CoV, while well-differentiated cells expressing more ACE2 were readily infected. ${ }^{10}$ ACE2 is less mature in young children and thus may not function properly as a receptor for SARS-CoV-2. ${ }^{11}$ In addition, the intracellular response induced by ACE2 in the alveolar epithelial cells of children may be lower than that of adults. ACE2 are more abundant on cells of the lower respiratory tract, ${ }^{12}$ which is typically the site of severe COVID-19 disease. Consistent with this observation, recent data indicate that children experience more SARS-CoV-2 infections in the upper respiratory tract than the lower respiratory tract. ${ }^{13}$

\section{Fetal Hemoglobin}

SARS-CoV-2 virus proteins (orf1ab, ORF10, and ORF3a) have been shown to attack the heme on the 1- $\beta$ chain of hemoglobin to dissociate the iron to form porphyrin. ${ }^{14}$ This attack not only reduces hemoglobin leading to hypoxia but also inhibits the normal metabolic pathway of heme. Liu and Li suggest that this mechanism moreover interferes with the normal heme anabolic pathway in the human body causing disease. Newborn infants have up to $80 \%$ fetal hemoglobin, made of alpha and gamma chain, which may be protective against the coronavirus. ${ }^{15}$ However, this explanation does 


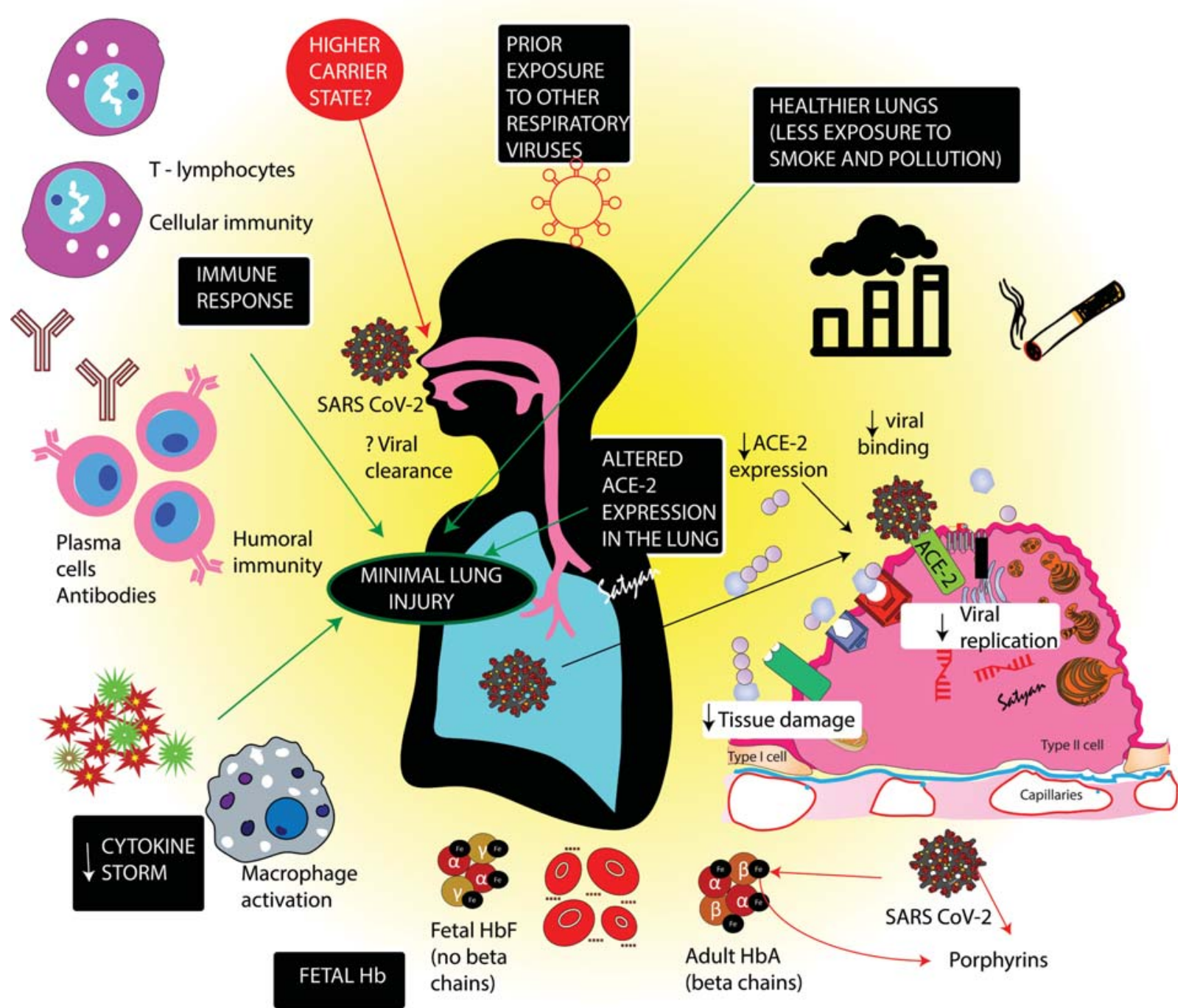

Fig. 2 A schematic showing possible factors resulting in low incidence and less severity of coronavirus disease (COVID-19) in pediatric age group. Children in generally have healthier lungs and are less exposed to smoke and pollution. The maturity, binding ability and function of Angiotensin converting enzyme (ACE2) receptors required by severe acute respiratory syndrome-coronavirus-2 (SARS-CoV-2) to enter the cells, is lower in children resulting in minimal lung injury. More extensive exposure to other viruses may provide cross immunity to SARS-COV2. A cytokine storm or systemic inflammatory response syndrome that results in inflammation and fluid buildup leading to respiratory distress is not well developed in children. More efficient T-cells response in children may be another reason for superior outcomes. SARS-CoV-2 proteins appear to attack $\beta$ hemoglobin chains and "capture" porphyrins inactivating gas exchange capabilities of hemoglobin $(\mathrm{Hb})$ and interfering with heme anabolic cycle. Young infants, with fetal $\mathrm{Hb}(\alpha 2 \gamma 2)$ without $\beta$ chains, may potentially be less susceptible to SARS-CoV-2 mediated effects on Hb. Image courtesy: Satyan Lakshminrusimha.

not explain the low incidence of severe disease in older children.

\section{Cross-Immunity with Other Viral Agents}

Acquired immunity with other viruses, including other coronaviruses, may be protective to the pediatric population. A study investigating the presence of short-lived relative crossprotection conferred by specific prior viral infections against subsequent febrile respiratory illness showed that adenoviruspositive participants tended to be protected against subsequent infection with adenovirus, coronavirus, enterovirus, rhinovirus, and influenza virus. ${ }^{16}$ On an average, children up to 6 years old can have 8 to 12 URIs per year in comparison to adolescents and adults who average 2 to 4 URIs per year. ${ }^{17}$ Severity of SARS$\mathrm{CoV}-2$ infection in infants with more likelihood of hospitaliza- tion as compared with toddlers and young children ${ }^{2}$ may be explained by the fact that infant's humoral immunity is initially dependent on maternal immunoglobulins.

\section{Obtunded Systemic Inflammatory Response Syndrome}

A cytokine storm or systemic inflammatory response syndrome is an overproduction of immune cells and their activating compounds associated with a surge of activated immune cells into the lungs resulting in inflammation and fluid buildup that can lead to respiratory distress and can be contaminated by a secondary bacterial pneumonia. This increases the risk of mortality in patients. ${ }^{18}$ This response is underdeveloped in children ${ }^{19}$ and could explain the recently published CDC data where only $54 \%$ pediatric cases had cough as compared with $80 \%$ in adults. ${ }^{20}$ Shortness of 
breath was also much lower in children compared with adults (13 vs. $43 \%){ }^{1}$ Clinical observations in Wuhan, China, have confirmed that the absolute number of peripheral blood lymphocytes in adult patients progressively declines during the inflammatory response to SARS-CoV-2, which could help the virus to proliferate and spread. The white blood cell count and the absolute number of lymphocytes in pediatric cases were found to be generally normal, which may be related to the incomplete development of natural immunity. ${ }^{2}$

\section{Differences in Humoral Immunity}

The use of convalescent serum containing neutralizing antibodies has been used previously in SARS-CoV and MERS and was recently reported in a small cohort for SARS-CoV-2. ${ }^{21}$ However, several studies imply certain antibody responses may contributed to pathology. ${ }^{22}$ In a SARS-CoV macaque model, antibodies against the Spike protein were shown to contribute to lung injury. In the feline infectious peritonitis virus, antibodies against the spike protein contributed to enhancement of infection. ${ }^{23}$ Higher neutralizing antibody titers against even SARS-CoV-2 have been correlated with lymphopenia and elevated C-reactive protein; however, this may simply represent increased immune stimulation from more severe illness. ${ }^{24}$ Because of differences in infectious history, children may use more favorable immunoglobulin isotypes or have improved antibody focused targeting to SARS-CoV-2.

\section{More Efficient T-cells}

Day et al. showed that T-cells are especially important in clearing viruses from mice infected with SARS-CoV. ${ }^{25}$ Another study in mice also stressed the importance of CD4+ helper T-cells, which stimulate B-cells to make antibodies against pathogens, in controlling SARS-CoV infection. ${ }^{26}$ In children, the young immune system and its efficient T-cells may potentially perform a superior job of responding to SARS-CoV-2. T-cell subsets also undergo dynamic changes between younger children and adolescents, particularly with declining $\mathrm{T}$ regulatory cells and increasing memory cells. $^{27}$

\section{Asymptomatic but Effective Carriers}

The incubation period of COVID-19 has been reported in the range of 0 to 24 days. ${ }^{28}$ Studies have shown asymptomatic and presymptomatic carrier transmission of the disease. ${ }^{29,30}$ In the United States, $27 \%$ of the pediatric cases that tested positive were asymptomatic, while only $7 \%$ of adults were asymptomatic as stated by CDC. An observational cohort study in Zhejiang, China, reported 28\% asymptomatic carriers among exposed children as well. ${ }^{31}$ Another study in Shenzhen, China, validated that children are at similar risk of infection as the general population, and less likely to have severe symptoms. ${ }^{32}$ These asymptomatic and mildly symptomatic children may play a major role in the spread of SARS-CoV-2 in the community and hence social distancing may be crucial to reduce the rate at which the pandemic spreads across the world. CDC recommends limiting a child's contact with older adults and people with chronic medical conditions.

\section{Conclusion}

Coronavirus leaves young children and neonates with minimal or no symptoms (a silver lining). This continued mystery may point to vital clues regarding both viral function and optimal immune system targeting. The milder and more often asymptomatic childhood presentations of SARS-CoV-2 may contribute to increased transmission of the virus to vulnerable adults (a black cloud). Studies in this area can help the researchers to develop prophylactic and therapeutic strategies to combat this deadly disease.

\section{Authors' Contributions}

M.R. drafted the manuscript and approved the final manuscript as submitted; P.C. conceptualized, edited, and approved the manuscript; M.D.H. reviewed, edited, and approved the final manuscript as submitted; S.L. mentored, reviewed, and approved the final manuscript as submitted. All authors approved the final manuscript as submitted and agree to be accountable for all aspects of the work.

\section{Note}

M.R., P.C.: American Academy of Pediatrics, Neonatal Resuscitation Program; Department of Pediatrics, University at Buffalo, Buffalo, NY. M.R.: Dr. Henry C. and Bertha H. Buswell Fellowship-Salary Support. M.D.H.: University at Buffalo, SUNY Research Seed Grant Program COVID-19.

\section{Conflict of Interest}

None declared.

\section{References}

1 CDC COVID-19 Response Team. Coronavirus disease 2019 in children-United States, February 12-April 2, 2020. MMWR Morb Mortal Wkly Rep 2020;69(14):422-426

2 Dong Y, Mo X, Hu Y, et al. Epidemiology of COVID-19 among Children in China. Pediatrics 2020:e20200702; Doi: 10.1542/ peds.2020-0702

3 Monto AS. Medical reviews. Coronaviruses. Yale J Biol Med 1974; 47(04):234-251

4 Center for Disease Control and Prevention. Weekly U.S. Influenza Surveillance Report. Available at: https://www.cdc.gov/flu/weekly/index.htm. Accessed April 8, 2020

5 Ferdinands JM, Olsho LE, Agan AA, et al; Pediatric Acute Lung Injury and Sepsis Investigators (PALISI) Network. Effectiveness of influenza vaccine against life-threatening RT-PCR-confirmed influenza illness in US children, 2010-2012. J Infect Dis 2014;210 (05):674-683

6 Stockman LJ, Massoudi MS, Helfand R, et al. Severe acute respiratory syndrome in children. Pediatr Infect Dis J 2007;26 (01):68-74

7 Li AM, Ng PC. Severe acute respiratory syndrome (SARS) in neonates and children. Arch Dis Child Fetal Neonatal Ed 2005; 90(06):F461-F465

8 Al-Tawfiq JA, Kattan RF, Memish ZA. Middle East respiratory syndrome coronavirus disease is rare in children: An update from Saudi Arabia. World J Clin Pediatr 2016;5(04):391-396

9 Zhou P, Yang XL, Wang XG, et al. A pneumonia outbreak associated with a new coronavirus of probable bat origin. Nature 2020;579 (7798):270-273 
10 Jia HP, Look DC, Shi L, et al. ACE2 receptor expression and severe acute respiratory syndrome coronavirus infection depend on differentiation of human airway epithelia. J Virol 2005;79(23): 14614-14621

11 Fang F, Luo XP. Facing the pandemic of 2019 novel coronavirus infections: the pediatric perspectives. Zhonghua Er Ke Za Zhi 2020;58(2):81-85

12 Hamming I, Timens W, Bulthuis ML, Lely AT, Navis G, van Goor H. Tissue distribution of ACE2 protein, the functional receptor for SARS coronavirus. A first step in understanding SARS pathogenesis. J Pathol 2004;203(02):631-637

13 Cruz AT, Zeichner SL. COVID-19 in children: initial characterization of the pediatric disease. Pediatrics 2020:e20200834

14 Wenzhong L, Li H. COVID-19: attacks the 1-beta chain of hemoglobin and captures the porphyrin to inhibit human heme metabolism. ChemRxiv 2020. Doi: 10.26434/chemrxiv.11938173.v7

15 Wong P, Weerakul J, Sritippayawan S. Hemoglobin analysis in the first year of life. Mediterr J Hematol Infect Dis 2016;8(01):e2016012

16 Chen IM, Loh JP, Chuah CXP, et al. Evidence for cross-protection against subsequent febrile respiratory illness episodes from prior infections by different viruses among Singapore Military Recruits 2009-2014. J Infect Dis 2019;219(12):1913-1923

17 Weintraub B. Upper respiratory tract infections. Pediatr Rev 2015; 36(12):554-556

18 Yang CS, Jinxiu Li, Jing Yuan, et al. Exuberant elevation of IP-10, MCP-3 and IL-1 ra during SARS-CoV-2 infection is associated with disease severity and fatal outcome. medRxiv 2020. Doi: 10.1101/2020.03.02.20029975

19 Jeljeli M, Guérin-El Khourouj V, Pédron B, Gressens P, Sibony O, Sterkers G. Ontogeny of cytokine responses to PHA from birth to adulthood. Pediatr Res 2019;86(01):63-70

20 Molloy EJ, Bearer CF. COVID-19 in children and altered inflammatory responses. Pediatr Res 2020

21 Shen C, Wang Z, Zhao F, et al. Treatment of 5 Critically Ill Patients With COVID-19 With Convalescent Plasma. JAMA 2020;323 (16):1582-1589
22 Liu L, Wei Q Lin Q et al. Anti-spike IgG causes severe acute lung injury by skewing macrophage responses during acute SARS-CoV infection. JCI Insight 2019;4(04):123158

23 Olsen CW, Corapi WV, Ngichabe CK, Baines JD, Scott FW. Monoclonal antibodies to the spike protein of feline infectious peritonitis virus mediate antibody-dependent enhancement of infection of feline macrophages. J Virol 1992;66(02):956-965

$24 \mathrm{Wu}$ F, Wang A, Liu M, et al. Neutralizing antibody responses to SARS-CoV-2 in a COVID-19 recovered patient cohort and their implications. medRxiv 2020. Doi: 10.1101/2020.03.30.20047365

25 Day CW, Baric R, Cai SX, et al. A new mouse-adapted strain of SARS-CoV as a lethal model for evaluating antiviral agents in vitro and in vivo. Virology 2009;395(02):210-222

26 Roberts A, Lamirande EW, Vogel L, et al. Animal models and vaccines for SARS-CoV infection. Virus Res 2008;133(01): 20-32

27 Shearer WT, Rosenblatt HM, Gelman RS, et al; Pediatric AIDS Clinical Trials Group. Lymphocyte subsets in healthy children from birth through 18 years of age: the Pediatric AIDS Clinical Trials Group P1009 study. J Allergy Clin Immunol 2003;112(05):973-980

28 Guan W-j, Ni Z-y, Hu Y, et al. Clinical characteristics of 2019 novel coronavirus infection in China. medRxiv 2020. Doi: 10.1101/ 2020.02.06.20020974

29 Bai Y, Yao L, Wei T, et al. Presumed asymptomatic carrier transmission of COVID-19. JAMA 2020;323(14):1406-1407

30 Chan JF, Yuan S, Kok KH, et al. A familial cluster of pneumonia associated with the 2019 novel coronavirus indicating person-toperson transmission: a study of a family cluster. Lancet 2020;395 (10223):514-523

31 Qiu H, Wu J, Hong L, Luo Y, Song Q, Chen D. Clinical and epidemiological features of 36 children with coronavirus disease 2019 (COVID-19) in Zhejiang, China: an observational cohort study. Lancet Infect Dis 2020:S1473-3099(20)30198-5

$32 \mathrm{Bi} \mathrm{Q}$ et al. Epidemiology and transmission of COVID-19 in Shenzhen China: analysis of 391 cases and 1,286 of their close contacts. medRxiv 2020. Doi: 10.1101/2020.03.03.20028423 